\title{
The Relations between Attention Deficit Hyperactivity Disorder and Headaches in a Non- Clinical Sample of Adolescents
}

\author{
Jacob Genizi ${ }^{1,2}$, Dana Marom ${ }^{1}$, Isaac Srugo ${ }^{1}$ and Nogah C Kerem ${ }^{1,3 *}$ \\ ${ }^{1}$ Pediatric Department, Bnai Zion Medical Center, Israel \\ ${ }^{2}$ Pediatric Neurology Unit, Bnai Zion Medical Center, Israel \\ ${ }^{3}$ Adolescent Medicine Unit, Bnai Zion Medical Center, Israel
}

Submission: September 24, 2016; Published: November 22, 2016

*Corresponding author: Nogah Kerem MD, Adolescent Medicine Unit, Bnai Zion Medical Center, Haifa, Israel, Tel: 972-4-8359662; Fax: 972-4-8359675; Email: nrkerem@hotmail.com

\begin{abstract}
Introduction: Stress is considered to be a major trigger for aggravation of headaches. In a previous study we demonstrated a high prevalence of ADHD among patients who were referred to a pediatric neurology clinic due to headaches. In the present study we examined whether this is true for a non-clinical population of adolescents.
\end{abstract}

Aims: To assess the prevalence of primary headaches among high-school students and to study their relations to ADHD.

Methods: Tenth grade high- school students in Haifa, Israel, anonymously filled a computerized questionnaire. The questionnaire contained questions about demographics, presence of headaches and their characteristics, and a previous diagnosis of ADHD.

Results: Out of 310 questionnaires, 230 students ( $81 \%$ ) reported experiencing headaches ( $88 \%$ of the girls and $76 \%$ of the boys, $p=0.08$ ), 110 of them (48\%) elaborated on the characteristics of their headaches: $50 \%$ matched migraine, $26 \%$ Tension Type Headache, and in $24 \%$ the data did not suffice for making a clear diagnosis of the headache type. Of the students who reported having headaches, $27 \%$ had a diagnosis of ADHD in comparison to $23 \%$ in those who did not report having headaches. Overall, no difference was found in ADHD prevalence between students who suffered from headache and those who didn't.

Conclusions: Our work indicates that ADHD is not a risk factor for headache among a non clinical sample of high- school students. Further research is needed.

Keywords: Migraine;Tension Type Headache; Attention deficit disorder

\section{Introduction}

Primary headaches are common in the pediatric population [1-3], predominantly migraine and tension type headaches (TTH) [4]. Since primary headaches may be disabling to children, several studies focused on the impact of headache on school performance [5-6], however the impact was reported to be minimal for most of the children [7]. Attention deficit hyperactivity disorder (ADHD) is also common among the pediatric population, and is considered to be very an important factor leading to poor academic performance. The worldwide prevalence of attention deficit disorder was shown to be $5.3 \%$ [8], and in the United States the prevalence was found to be higher, at $8 \%$ for children aged 8 to 15 years [9]. Although the association between primary headaches and emotional and behavioral problems in children has been previously described [10-11], the prevalence of ADHD among children with headaches as well as their association with headache duration and frequency remains unclear. It may be due to the fact that many children with ADHD have emotional disorders such as anxious disorders or depressive disorder, as a co morbid diagnosis. Numerous studies in adults with migraine, showed impairment in tasks such as psychomotor speed, attention, language [12-13] and executive functions [14], while others failed to confirm these findings [15]. In adults with tension type headache no cognitive dysfunction was identified [16].

Numerous psychological predictors for the emergence of headaches in the pediatric age group were identified, among them: concentration difficulties, emotional rigidity, deliberation, 
hyperactivity and stress among the family or at school [17-18]. On the other hand, frequent headaches may increase distractibility, in any child, but particularly in children with primary short attention span and thus may hold a negative impact on school work and performance. In a previous study [3] we have demonstrated an association between headaches, ADHD and school achievements among children who were referred to a pediatric neurology clinic for headaches. However, it was found that children who had headaches in addition to another complaint (somatic complaint) were more likely to be referred to a pediatric neurology clinic than were children who only complained of headaches [19], therefore it was questionable whether the conclusions of our previous study could be implied to a more general pediatric population. To overcome this potential bias we performed the current study, which was conducted in a high- school setting, therefore with a non- clinical sample of students. The purpose of the present study was to assess the prevalence of primary headaches among highschool students and to study the relations between headache presence, type of headache and ADHD.

\section{Method and Patients}

Study design - Cohort study, transversal, observational.

Patients -10th grade high- school students attending schools in Haifa, Israel. All gave informed assents to the study and their parents gave an informed consent via e- mail. There were no exclusion criteria other than not consenting to participation in the study.

Methods - Students anonymously filled a computerized general health questionnaire, that was designed for assessing the prevalence of headaches among high- school students and its' associations with other comorbidities and risk taking behaviors. The questionnaire included questions about demographics, whether students had headaches or not (and if they had - their characteristics, including age at onset, location, quality, frequency, duration, aura, associating symptoms, and family history). It also included questions about ADHD (previous diagnosis, related symptoms, medications recommended and taken), school achievements, risk taking behaviors and other known diagnoses (physical and psychological alike). The prevalence of headaches and their types was assessed, and diagnosis of tension type headache or migraine was made according to the international classification of headache disorders ( $2^{\text {nd }}$ edition) [20]. Headache association with ADHD was then assessed.

Statistical analysis - Data were summarized as proportions or means and standard deviations. Bivariate analyses of the associations between headache diagnosis, frequency and duration of headache and background factors (gender, learning disorders, attention deficit disorder and school functioning) were conducted with Mann-Whitney $U$ tests. Multivariate analyses of the associations between headache diagnosis and learning disorders, attention deficit and school functioning, were conducted with nominal regressions. Multivariate analyses of the associations between frequency and duration of headache and learning disorders, attention deficit disorder and school functioning, were conducted with logistic regressions. Analyses for the total sample, in both nominal and logistic regressions, were adjusted for gender. $P$ value of $\leq 0.05$ was considered as statistically significant.

\section{Results}

310 questionnaires were filled by tenth grade students (ages 15-16), 279 were completed and analyzed, of which 158 (57\%) were boys and $121(43 \%)$ girls. 230 students (81\%) complained about headaches $(88 \%$ of the girls and $76 \%$ of the boys, $\mathrm{p}=0.08$ ). However only 110 students (48\%), 47 boys and 63 girls, gave a full description of the characteristics of their headaches, so a diagnosis was feasible according to the international classification of headache disorders (2nd edition) [21]. Out of those, 55 students (50\%) matched a diagnosis of migraine, 42 of them (76\%) without aura and 13 (24\%) with aura. Males and females had the same prevalence of migraine ( $47 \%$ of the males, $52 \%$ of the females). 29 students (26\%) matched a diagnosis of Tension Type Headache, without difference between the genders $(\mathrm{p}=0.13)$. In 26 students (24\%) headache characteristics did not match any primary headache diagnosis ( $19 \%$ of the males, $27 \%$ of the females).

Among the students who reported to have headaches: $27 \%$ were diagnosed as having ADHD, compared to $22.5 \%$ in the group that didn't have headaches, no significant statistical difference was found. No difference was found in headache prevalence among students with ADHD between those taking medications such as methylphenidate and those not.

\section{Discussion}

The present study demonstrates a high rate (81\%) of adolescents that complain about headaches. Our findings are similar to the published data concerning pediatric headache $[3,21]$ including a higher rate among girls (88\% VS 76\%) [22]. According to the international classification of headache disorders [20] headache description matched migraine in about $50 \%$ of the students, with a proceeding Aura in $24 \%$ of the cases with no difference between boys and girls. These findings do not match data collected from other studies, done with clinical samples of children and adolescentsv [3,23]. These studies described a higher rate of migraine among girls. The rate of TTH in our study was relatively low (26\%), with a high percentage of cases (24\%) in which the description of headache did match any primary headache diagnosis.

A high rate of ADHD (26\%) was found in our study. Leviton [19] was one of the first to report about the relation between headaches and academic difficulties: he reported that out of 150 elementary-school children who were referred to his clinic due to recurrent headaches, approximately $40 \%$ also had academic difficulties. The high prevalence of ADHD found in our study is in accordance with other recent studies, which found a high 
incidence of hyperactivity and impulsivity symptoms in children with headache, when compared with their peers [11,24]. In a population- based study on healthy 4 to 17 year-old children, done by Strine et al. [10], children with frequent headaches were 2.6 times more likely to have inattention and hyperactivity.

A previous study we have conducted also indicated a high rate of ADHD and learning disabilities in children referred to pediatric neurology clinics due to recurrent episodes of headaches: $28 \%$ of them had ADHD, 24.7\% had learning disabilities, and $12 \%$ had both [3].

In our present study we found no significant differences in the prevalence of ADHD between the students having headaches and those who did not. Two possible explanations to that are the relatively small sample size, and the inclusion of those who had infrequent episodes of headache in the "headache group". A significant difference was found between students with and without headache in relation to a subjective report of having symptoms of ADHD and learning disabilities, without having a formal diagnosis of these disorders: Those with headaches had a much higher rate of this unpleasant notion than their peers who did not report having headaches. These findings may be explained from two standpoints: The first is that significant headaches could interrupt with school work and hold a negative impact on academic achievements, as was shown in a recent study [25]. Hence, those students who have headaches and do not succeed may search for an "objective barrier" for being unsuccessful, such as having ADHD or a learning disability, even when a formal assessment was not done. From the other hand, stress in the school setting was shown to be a contributor for headaches in children and adolescents. Therefore those students who experience difficulties with school work, but do not "hold a title" that enables them to be more comfortable with their difficulties (such as ADHD or learning disabilities) might feel more stressed than their peers who have formal diagnoses, hence receive assistance and probably more acceptance of their difficulties from their teachers/ peers / parents. Those students may have a higher level of stress, and therefore may be more prone to having headaches. This is in accordance with Strine's report that children who exhibit high level of emotional, attention, language and peer problems, were significantly more likely to have their difficulties interfere with classroom learning and leisure activities [10].

The subjects in our study were high- school students, and not adolescents who seek for medical advice in a clinical setting. The choice of a non- clinical sample of subjects has an advantage in evaluating the correlation between two symptoms as it overcomes the "Berkson's principle". Joseph Berkson demonstrated [26] that people, who seek medical care, are more likely to have more than one medical problem. Therefore, the relationship of two diseases should not be studied in those who seek for medical care, according to Berkson's principle. The choice of studying a non- clinical sample in the current study versus studying patients who were referred to a neurology clinic due to headaches in our previous study could explain the differences in the prevalence of ADHD found between the two studies. Using a computerized survey for assessing health and behavioral challenges in adolescents was previously shown to be a valid method in numerous studies [27-28].

\section{Limitations}

A computerized survey has its disadvantages due to the lack of personal communication that may allow further explanations in order to avoid misinterpretations either by the students filling the questionnaire, or by the investigators analyzing it. This possible misinterpretation of some questions by the students might be a contributor for the relatively high percentage of students (24\%) that the description of their headache did not match any primary headache diagnosis, and to the relatively high rate of students reporting they were diagnosed with ADHD. It should also be noted that headache as a somatic complaint could be a part of emotional status and we didn't look at the student's emotional status as well as at sleep disorder and others ADHD comorbidities.

\section{Conclusion}

Our study demonstrated that headache is a frequent complaint among high school students, and that ADHD was not more frequent among students suffering from headaches when compared to their peers who did not report having headache episodes. However, students with learning difficulties but without a formal diagnosis of ADHD reported more headaches, possibly due to an elevated stress level, that might lead to a higher rate of headache episodes.

\section{References}

1. Stovner LJ, Hagen K, Jensen R, Katsarava Z, Lipton R, et al. (2007) The global burden of headache: a documentation of headache prevalence and disability worldwide. Cephalalgia 27(3): 193-210.

2. Laurell K, Larsson B, Eeg-Olofsson O (2004) Prevalence of headache in Swedish schoolchildren, with a focus on tension-type headache. Cephalalgia 24(5): 380-388.

3. Genizi J, Gordon S, Kerem NC, Srugo I, Shahar E, et al. (2013) Primary headaches, attention deficit disorder and learning disabilities in children and adolescents. J Headache Pain 14(1): 54.

4. Zwart JA, Dyb G, Holmen TL, Stovner LJ, Sand T, et al. (2004) The prevalence of migraine and tension-type headaches among adolescents in Norway. The Nord-Trondelag Health Study (Head-HUNT-Youth), a large population based epidemiological study. Cephalalgia 24(5): 373379.

5. Ando N, Fujimoto S, Ishikawa T, Teramoto J, Kobayashi S, et al. (2007) Prevalence and features of migraine in Japanese junior high school students aged 12-15 yr. Brain Dev 29(8): 482-485.

6. Akyol A, Kiylioglu N, Aydin I, Erturk A, Kaya E, et al. (2007) Epidemiology and clinical characteristics of migraine among school children in the Menderes region. Cephalalgia 27(7): 781-787.

7. Bille B (1997) A 40-year follow-up of school children with migraine. Cephalalgia 17(4): 488-491.

8. Polanczyk G, Lima MSD, Horta BL, Biederman J, Rohde LA, et al. (2007) The worldwide prevalence of ADHD: a systematic review and metaregression analysis. Am J Psychiatry 164(6): 942-948. 
9. Froehlich TE, Lanphear BP, Epstein JN, Barbaresi WJ, Katusic SK, et al (2007) Prevalence, recognition, and treatment of attention-deficit/ hyperactivity disorder in a national sample of US children. Arch Pediatr Adolesc Med 161(9): 857-864.

10. Strine TW, Okoro CA, McGuire LC, Balluz LS (2006) The associations among childhood headaches, emotional and behavioral difficulties, and health care use. Pediatrics 117(5): 1728-1735.

11. Mazzone L, Vitiello B, Incorpora G, Mazzone D (2006) Behavioral and temperamental characteristics of children and adolescents suffering from primary headache. Cephalalgia 26(2): 194-201.

12. Hooker WD, Raskin NH (1986) Neuropsychologic alterations in classic and common migraine. Arch Neurol 43(7): 709-712.

13. Calandre EP, Bembibre J, Arnedo ML, Becera D (2002) Cognitive disturbances and regional cerebral blood flow abnormalities in migraine patients: Their relationship with the clinical manifestations of the illness. Cephalalgia 22(4): 291-302.

14. Mongini F, Keller R, Deregibus A, Barbalonga E, Mongini E, et al. (2005) Frontal lobe dysfunction in patients with chronic migraine: A clinicalneuropsychological study. Psychiatry Res 133(1): 101-106.

15. Pearson AJ, Chronicle EP, Maylor EA, Bruce LA (2006) Cognitive function is not impaired in people with a long history of migraine: A blinded study. Cephalalgia 26(1): 74-80.

16. Waldie KE, Welch D (2007) Cognitive function in tension type headache. Curr Pain Headache Rep 11(6): 454-460.

17. Aromaa M, Rautava P, Helenius H, Silanpaa M (1998) Factors of early life as predictors of headache in children at school entry. Headache 38(1): 23-30.

18. Carlsson J (1996) Prevalence of headaces in schoolchildren: relation to family and school factor. Acta Paediatr 85(6): 692-696.
19. Leviton A (1986) Do learning handicaps and headache cluster? J Child Neurol 1(4): 372-377.

20. (2004) Headache Classification Committee of the International Headache Society. The international classification of headache disorders ( $2^{\text {nd }}$ edn), Cephalalgia 24: 9-160.

21. King S, Chambers CT, Huguet A, MacNevin RC, McGrath PJ, et al. (2011) The epidemiology of chronic pain in children and adolescents revisited: A systematic review. Pain 152(12): 2729-2738.

22. Genizi J, Srugo I, Kerem NC (2013) The cross- ethnic variations in the prevalence of headache and other somatic complaints among adolescents in Northern Israel. J Headache Pain. 14(1): 21.

23. Abu-Arefeh I, Russell G (1994) Prevalence of headache and migraine in schoolchildren. BMJ 309(6957): 765-769.

24. Arruda MA, Guidetti V, Galli F, Albuquerque RC, Bigal ME, et al. (2010) Migraine, tension-type headache, and attention-deficit/hyperactivity disorder in childhood: a population-based study. Postgrad Med 122(5): 18-26.

25. Kantor D (2012) The impact of migraine on school performance. Neurology 79(18): e168-e169.

26. Berkson J (1946) Limitations of the application of fourfold table analysis to hospital data. Biometrics 2(3): 47-53.

27. Supple AJ, Aquilino WS, Wright DL (1999) Collecting Sensitive Self-Report Data with Laptop Computers: Impact on the Response Tendencies of Adolescents in a Home Interview. Journal of Research on Adolescence 9(4): 467-488.

28. Webb PM, Zimet GD, Fortenberry JD, Blythe MJ (1999) Comparability of a computer-assisted versus written method for collecting health behavior information from adolescent patients. Journal for Adolescent Health 24(6): 383-388.

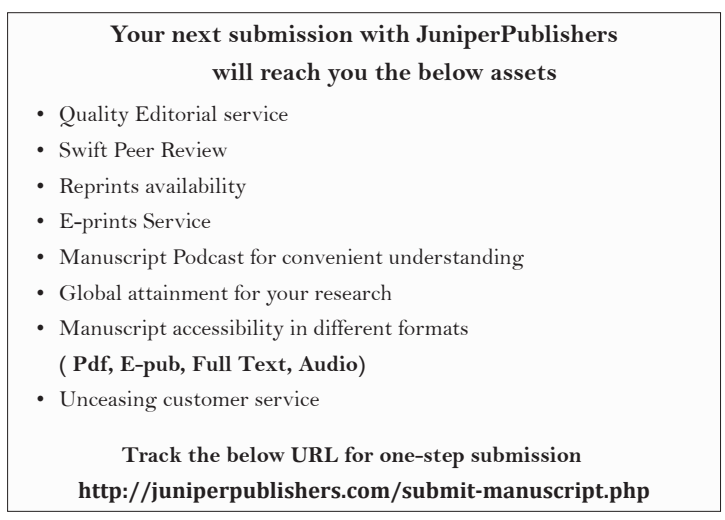

